\title{
Ascites do not affect the rate of complete cytoreductive surgery and prognosis in patients with primary ovarian cancer with ascites treated with hyperthermic intraperitoneal chemotherapy
}

\author{
MINGCHEN BA ${ }^{1}$, HUI LONG ${ }^{2}$, XIANGLIANG ZHANG ${ }^{1}$, ZHAOFEI YAN ${ }^{1}$, \\ SHUAI WANG ${ }^{1}$, YINBING WU ${ }^{1}$, YUANFENG GONG ${ }^{1}$ and SHUZHONG CUI ${ }^{3}$ \\ ${ }^{1}$ Intracelom Hyperthermic Perfusion Therapy Center, Cancer Hospital and Institute of Guangzhou Medical University; \\ ${ }^{2}$ Guangzhou Dermatology Institute, Guangzhou, Guangdong 510095; \\ ${ }^{3}$ Guangzhou Baorui Medical Technology Co., Ltd. Guangzhou, Guangdong 510540, P.R. China
}

Received March 16, 2016; Accepted August 6, 2018

DOI: $10.3892 / \mathrm{ol} .2019 .10493$

\begin{abstract}
Cytoreductive surgery (CRS) is the current standard therapy procedure for patients with advanced ovarian cancer (OC), but numerous patients with OC are complicated with ascites. The aim of the present study was to assess whether massive ascites affect the rate of complete CRS and prognosis for patients with primary $\mathrm{OC}$ treated with hyperthermic intraperitoneal chemotherapy (HIPEC). Between December 2006 and December 2015, 1,293 patients with primary OC from the Intracelom Hyperthermic Perfusion Therapy Center of the Cancer Hospital of Guangzhou Medical University prospective database were treated with CRS combined with HIPEC. A total of 1,225 patients were without malignant ascites or small amounts of ascites and 68 had massive malignant ascites. The rate of complete CRS, overall survival (OS), disease-free survival (DFS) and resolution of ascites for patients with massive ascites were analyzed between patients without/small ascites, and with massive ascites. Complete CRS was successful in $86.8 \%(1,063 / 1,225)$ of patients without/small ascites, and $85.3 \%$ (58/68) of patients with massive ascites. No statistical differences were identified in complete CRS success between patients with ascites and patients without/small ascites $(\mathrm{P}=0.080)$. For patients with massive ascites, all symptoms exhibited regression; the total objective remission rate was
\end{abstract}

Correspondence to: Dr Mingchen Ba, Intracelom Hyperthermic Perfusion Therapy Center, Cancer Hospital and Institute of Guangzhou Medical University, 78 Hengzhigang Road, Guangzhou, Guangdong 510095, P.R. China

E-mail: bamingchen0713@163.com

Dr Hui Long, Guangzhou Dermatology Institute, 56 Hengfu Road, Guangzhou, Guangdong 510095, P.R. China

E-mail: 798587414@qq.com

Key words: cytoreductive surgery, hyperthermic intraperitoneal perfusion chemotherapy, primary ovarian cancer, malignant ascites, peritoneal carcinomotosis
$100 \%$ (68/68), even for patients with incomplete CRS (10/68) $(\mathrm{P}=0.100)$. The mean OS was 58 months and the mean DFS was 26 months in patients without/small ascite, vs. 57 months and 28 months in patients with massive ascites. No significant differences were noted in median DFS and median OS between patients with ascites, and patients without/small ascites (All $\mathrm{P}>0.05$ ). In conclusion, the results of the present study suggest that ascites does not affect the rate of complete CRS and the prognosis of patients with massive ascites following HIPEC. CRS is suitable for the majority of patients with primary OC and massive ascites.

\section{Introduction}

Ovarian cancer $(\mathrm{OC})$ is the leading cause of mortality among all gynecological cancer types in developed countries and the fifth leading global cause of mortality from cancer in women (1). Due to the lack of clinical signs and the absence of effective screening tests, $70-80 \%$ of patients are diagnosed at the advanced stages, when peritoneal carcinomatosis (PC) is established, and numerous cases are complicated with ascites $(1,2)$. Malignant ascites frequently occur in patients $\mathrm{OC}$ when PC is present, in which fluid containing cancer cells accumulate in the abdominal cavity (3-7). With the increasing amount of ascites, discomforts and decreased quality of life (QOL) associated with symptomatic malignant ascites often exceed that of the cancer itself, resulting in detrimental physiological, and psychological states leading to poor prognosis (4-9).

The standard of care for the treatment of advanced OC includes optimal cytoreductive surgery (CRS) with adjuvant taxanes- and platinum-based chemotherapy $(1,2,10,11)$. However, patients with primary $\mathrm{OC}$ and massive ascites present highly variable health conditions, and certain patients with OC and massive ascites are not eligible for CRS due to poor health conditions and unstable vital signs (4). The authors of the present study previously reported that the efficacy of CRS decreased with increasing amounts of ascites in patients with extensive PC from gastric and colon cancer (4-7). In addition, a retrospective study suggested that the presence of malignant ascites significantly decreases the chances of achieving a complete CRS (3). 
Hyperthermic intraperitoneal chemotherapy (HIPEC) has been demonstrated to be a promising treatment for PC and malignant ascites (3-9). Maximum CRS according to the Sugarbaker criteria (12) combined with HIPEC have achieved good clinical efficacy for improving QOL $(13,14)$ and prolonging the survival of carcinomatosis of non-ovarian origins, including the stomach (15-18), colon $(19,20)$, and appendix $(21,22)$. CRS may be used to remove bulky tumor tissue (10,11,23-29), and HIPEC may then be used to eradicate residual microscopic tumors in the peritoneal cavity (30-38), thereby improving the QOL $(13,14)$.

Unlike peritoneal diffuse metastases from advanced digestive tract carcinomatosis, whereby CRS result in few changes, $\mathrm{PC}$ in the majority of patients with $\mathrm{OC}$ is due to local infiltrative metastases or limited regional peritoneal metastases (39-41). Therefore, the complete CRS success rate for these patients should be considerably higher compared with the rates reported for patients with digestive tract tumors and malignant ascites $(3,6)$.

Despite favorable response rates to this combined approach, numerous patients are not eligible due to poor health conditions $(3,4,42)$. Consequently, novel approaches to HIPEC should be considered if oncologists are to achieve better prognoses in their patients. HIPEC by minimally invasive or non-invasive surgical approaches, including B-mode ultrasound-guided placement of perfusion catheters, have recently been proposed for patients who are not eligible for CRS (4-6).

Although CRS with HIPEC has been demonstrated to exhibit more benefits in patients with OC carcinomatosis compared with non-ovarian carcinomatosis (4-7), it has not yet been determined if massive ascites, particularly in patients with poor health conditions, affect the complete CRS rate and prognosis of patients with primary OC under HIPEC. Therefore, the aim of the present study study was to evaluate the possibility of complete CRS and prognosis for primary OC complicated with massive ascites using the prospective database of patients with primary OC treated with HIPEC at the Intracelom Hyperthermic Perfusion Therapy Center of the Cancer Hospital of Guangzhou Medical University (Guangzhou, China).

\section{Patients and methods}

Study design. The present study was a retrospective study performed using a prospective cohort registered at the Intracelom Hyperthermic Perfusion Therapy Center of the Cancer Hospital of Guangzhou Medical University. Patients were treated between December 2006 and December 2014. Patients with primary OC were included. The presence of massive ascites was determined by ultrasound or other imaging modalities. According to their general health condition prior to treatments, patients with massive ascites were divided into the good or the poor condition groups. The patients in a good condition were treated with CRS followed by HIPEC (CRS+HIPEC). The patients in a poor condition were treated using B-mode ultrasound-guided HIPEC first followed by delayed CRS upon general condition improvement (dCRS+HIPEC). Patients were considered to be in poor condition if the following applied: i) Heart rate, $>100 \mathrm{bpm}$ at rest; ii) respiration rate, $>20$ breaths/min at rest; and iii) blood oxygen saturation, $<95 \%$ at rest. Otherwise, the patients were included in the good condition group. All patients with primary OC were attempted to be treated with CRS combined with HIPEC first. Patients with small ascites and those without ascites were treated in the same way as patients in the good condition group (immediate CRS followed by HIPEC during CRS).

The inclusion criteria were: i) $\geq 18$ years old; ii) diagnosis of primary OC; iii) no radiation therapy in the previous 4 weeks; iv) no chemotherapy in the previous 2 weeks; and v) the tumor board at the Cancer Hospital of Guangzhou Medical University determined that the prognosis was $>2$ months. The exclusion criteria were as follows: i) Recurrence of $\mathrm{OC}$ with ascites; ii) known or possible ovarian metastases from other organs; iii) known or possible primary malignant tumor in other internal organs; iv) known or potential pregnancy; or v) extensive abdominal adhesions due to multiple surgeries. The present study was approved by the Medical Ethics Committee of the Cancer Hospital of Guangzhou Medical College (approval no. GZYY2006-8-20). Written informed consent was obtained from all patients.

CRS and placement of perfusion catheters. For CRS, all patients were treated with the first intention of achieving complete CRS. Patients with massive ascites in the good condition group and patients without/small ascites were treated with immediate CRS followed by HIPEC. Patients in the poor condition group were treated with dCRS following the improvement of their general health condition after HIPEC.

Surgery was performed under general anesthesia and endotracheal intubation. For patients with massive ascites, after opening the abdominal wall, $200 \mathrm{ml}$ of ascites were sent for cytological examination; then, all remaining abdominal fluid was suctioned. CRS consisted of the removal of all gross tumors and involved organs, peritoneum or tissue, as deemed technically feasible, and safe for the patient. Any tumors adhering to or invading vital structures that could not be removed were cytoreduced using a cavitational ultrasonic surgical aspirator (Valleylab ${ }^{\mathrm{TM}}$; Medtronic, Minneapolis, MN, USA).

The International Federation of Gynecology and Obstetrics classification for OC staging was used (42). The anatomical extension of the $\mathrm{OC}$ in the peritoneal cavity was best evaluated by the PC index (PCI), as described by Sugarbaker (43). The resection status was determined after CRS using the following classification: R0, complete removal of all visible tumor and negative cytological findings or microscopic margins; R1, complete removal of all visible tumor and positive post perfusion cytologic findings or microscopic margins; R2a, minimal residual tumor, nodule(s) measuring $\leq 5 \mathrm{~mm}$; $\mathrm{R} 2 \mathrm{~b}$, gross residual tumor, nodule(s) measuring $>5 \mathrm{~mm}$, but $\leq 2 \mathrm{~cm}$; and R2c, extensive disease remaining, nodule(s) measuring $>2$ $\mathrm{cm}$. According to these, R0/R1 resections were considered as complete CRS, while $\mathrm{R} 2 \mathrm{a}-\mathrm{R} 2 \mathrm{c}$ resections were considered as incomplete CRS (43).

Following CRS, an infusion catheter with multiple side holes (inner diameter of $0.8 \mathrm{~cm}$, outer diameter of 1.0 and $100 \mathrm{~cm}$ in length) was placed into the peritoneal cavity in the upper left and right quadrant with $40-60 \mathrm{~cm}$ of catheter 
inside the body (Fig. 1). Similarly, an outflow catheter with the same dimensions was placed in the lower left and right quadrant of the pelvic cavity. The abdominal wall was sutured and the perfusion catheter was fixed to the abdominal wall by cutaneous sutures, as previously described (4).

B-mode ultrasound-guided placement of catheters for chemotherapy (Fig. 2). For patients in the poor condition group, those who could not tolerate endotracheal general anesthesia and CRS, B-mode ultrasound-guided HIPEC was performed first, and dCRS was performed after improvement of their general condition.

In a standard operating room, patients were placed in the supine position. Pethidine hydrochloride (75 mg; Qinghai Pharmaceutical Factory Co., Xining, China) and promethazine hydrochloride $(25 \mathrm{mg}$; Shuntong Pharmaceuticals (Shuntong, China) were administered by intramuscular injection. Propofol (Astra Zeneca, London, UK) was given intravenously in a continuous manner at a dosage rate of $3-8 \mathrm{ml} / \mathrm{h}$; this was adjusted according to the patient's status. B-ultrasound examination of all four abdominal quadrants was performed to select the best puncture location. The region with the largest amount of ascites that were not adhering to the abdominal wall or to the tissues of the peritoneal cavity was selected. Additionally, the region had to be without previous abdominal incision or tumor. A 1.2-cm incision was made after locally administering $0.5 \%$ lidocaine and a Hasson trocar $(1.2 \mathrm{~cm}$ in diameter) was placed into the peritoneal cavity. Then, the infusion and outflow catheters with multiple side holes (inner diameter of $0.8 \mathrm{~cm}$, outer diameter of 1.0 and $100 \mathrm{~cm}$ in length) were placed into the intraperitoneal cavity. The infusion catheters were positioned in the left and right upper quadrants of the intraperitoneal cavity with an inside length of 40-80 $\mathrm{cm}$. The outflow catheters were placed in the pelvic cavity of the left and right lower quadrants with the same length as the infusion catheters. The ascites were extracted as completely as possible. All perfusion catheters were fixed to the abdominal wall by cutaneous sutures, as previously described (4).

HIPEC. HIPEC was performed using our self-developed 'BR-TRG-I type high-precision hyperthermic intraperitoneal perfusion treatment system' (BR-TRG-I; Guangzhou Baorui Medical Devices Ltd. Company, Guangzhou, China; www. gzbrm.com), as previously described (4-7) (Fig. 3). This device, the only one of its kind, was approved for use by the State Food Drug Administration Firearms of China (approval no. 2009-3260924).

The treatment temperature during HIPEC was measured using the BR-TRG-I treatment system with temperature-monitoring probes at several locations: In the infusion and outflow catheters; and at the peritoneal surface of pelvic cavity. The vital signs of patients (blood pressure, heart rate, respiratory rate and blood oxygen saturation) were assessed using the 'multi-parameter patient-monitoring machine' (G3HJ20025; Shenzen Mindray Bio Medical Electronics Co.,Ltd., Shenzhen, China).

HIPEC therapy consisted of three sessions. First, 0.9\% saline solution or $5 \%$ glucose, with a volume $500 \mathrm{ml}$ greater than the cavity $(3,000-7,000 \mathrm{ml})$ was added to the custom infusion bag and delivered via the infusion tubes over 90 min with a

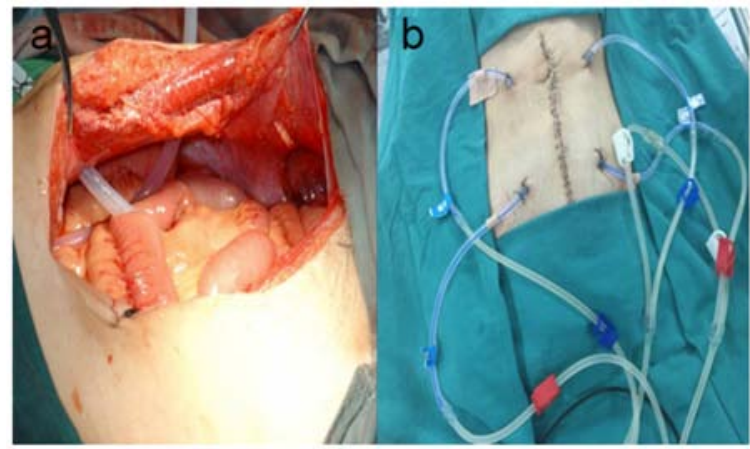

Figure 1. Placement sites of infusion catheters and outflow catheters for HIPEC during CRS surgery. (A) Placement sites of the infusion and outflow catheters in peritoneal cavity during CRS surgery. (B) Placement sites of the infusion and outflow catheters on peritoneal wall during CRS surgery. CRS cytoreductive surgery; HIPEC, hyperthermic intraperitoneal chemotherapy.

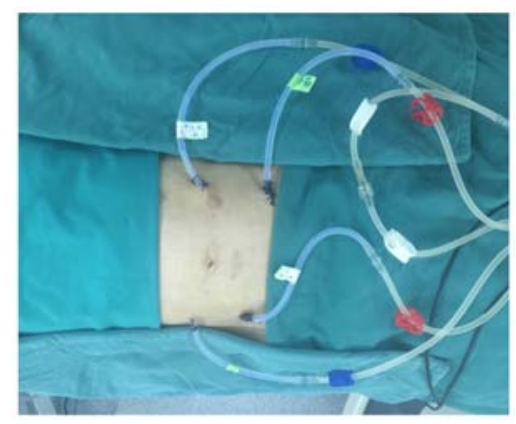

Figure 2. Placement sites of the infusion and outflow catheters for B-mode ultrasound-guided HIPEC HIPEC followed by dCRS. Placement sites of the infusion and outflow catheters on peritoneal wall $\mathrm{d}$ for B-mode ultrasound-guided HIPEC HIPEC followed by dCRS. The red clips mark the two infusion catheters. The blue clips mark the two outflow catheters. The white clips mark the loop circuit for HIPEC preparation. dCRS, delayed cytoreductive surgery; HIPEC, hyperthermic intraperitoneal chemotherapy.

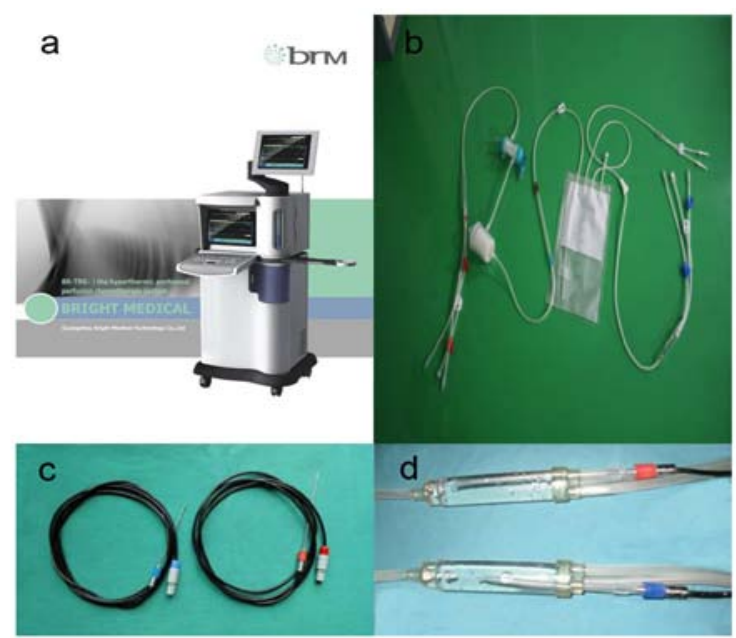

Figure 3. The BR-TRG-I type-high precision hyperthermic perfusion intra-peritoneal treatment system and pipeline system. (A) The BR-TRG-II type-high precision hyperthermic perfusion intra-peritoneal treatment system; (B) pipeline system; and (C) temperature-monitoring probes. One tip was put into infusion catheters or outflow catheters and one tip was connected to the 'BR-TRG-I type high-precision hyperthermic intraperitoneal perfusion treatment system'. (D) Temperature-monitoring probe locations, red cap mark location in the infusion catheters and blue cap mark location in the outflow catheters. BR-TRG-II, BR-TRG-II type high-precision hyperthermic intraperitoneal perfusion treatment system. 
velocity of $450-600 \mathrm{ml} / \mathrm{min}$, and an in-flow temperature of $43^{\circ} \mathrm{C}$ to achieve an interior abdominal temperature of $41.5-42.5^{\circ} \mathrm{C}$. The hyperthermic intraperitoneal chemotherapeutic agents added into the perfusion fluid were docetaxel $75 \mathrm{mg} / \mathrm{m}^{2}$ (Jiangsu Aosaikang Pharmaceutical Co., Ltd., Nanjing, China) for the first session and cisplatin $80 \mathrm{mg} / \mathrm{m}^{2}$ (Jiangsu Haosen Pharmaceutical Ltd., Nanjing, China, http://www.hspharm. $\mathrm{cn} / \mathrm{en} /$ ) for the second and third sessions. After the third HIPEC session, all ascites was drained out and the infusion catheter was removed. The outflow catheter was kept for 3-5 days as a closed drainage catheter.

Follow-up. All patients were followed up till July 2015 or mortality. Follow-up was performed at 1 and 3 months, and then every 3-6 months thereafter for up to 1 year. After 1 year, follow-up was performed at 6-month intervals or less frequently if the patient continued to remain without evidence of disease. Abdominal and pelvic computed tomography were obtained at 3, 6 and 12 months following treatment or when clinically indicated. All patients received systemic chemotherapy using paclitaxel and cisplatin at the discretion of their oncologist.

Remission from ascites and tumor progression was classified into three grades according to our previously modified World Health Organization criteria on efficacy assessment in malignant tumors: i) Complete remission (CR): Ascites are completely absorbed after treatment, which is sustained for at least 4 weeks; ii) partial remission (PR): Ascites are reduced by $50 \%$, which is sustained for at least 4 weeks; iii) no consequence (NC): Ascites are not reduced obviously or increased within 4 weeks after treatment (4-7). The Eastern Cooperative Oncology Group performance status and Karnofsky performance score (KPS score) were used to evaluate ascite remission, and patient QOL $(5,6)$. Objective remission rate (ORR) for assessment remission of ascites, and the KPS score for measure of functional impairment was performed on day 1 at the beginning of CSR and HIPEC treatment, and at 4 weeks post-completion of all CSR and HIPEC treatment. Overall survival (OS) was defined as the time between CRS+HIPEC or dCRS+HIPEC completion and mortality. Disease-free survival (DFS) was defined as the time between HIPEC or dCRS+HIPEC completion and the recurrence of PC. Data from the 4-week follow up after HIPEC+CRS were recorded and used in statistical analyses. All patients were monitored until mortality or December 2015.

Statistical analysis. Data were analyzed using SPSS 17.0 (IBM Corp., Armonk, NY, USA). All continuous data are presented as mean \pm standard deviation and were analyzed using the Student's t test (between groups) or the paired $t$ test (before/after therapy). Categorical data are presented as frequencies and were analyzed using the chi-square test for categorical variables and the independent two-way analysis of variance (ANOVA) for continuous variables. Multiple group comparison was conducted by one-way ANOVA followed by the Bonferroni correction. OS and DFS were analyzed, and compared using the Kaplan-Meier estimator method. Differences in survival between groups were determined by the log-rank test. $\mathrm{P}<0.05$ was considered to indicate a statistically significant difference.

\section{Results}

Characteristics of patients. A total of 1,293 female patients with OC treated with CRS+HIPEC were included in the current study, which included 1,225 patients (mean age, $54.3 \pm 2.6$ years; range, $33-77$ years) without/small ascites, and 68 patients (mean age, $54.0 \pm 2.9$ years; range, 30-79 years) with massive ascites. Age was comparable between patients without/small ascites and patients with massive ascites $(\mathrm{P}=0.100)$. The frequency of massive ascites was $5.3 \%$ in patients with OC in the present study.

CRS and HIPEC during CRS (CRS+HIPEC) were performed in 1,225 female patients without/small ascites and in 46 patients with massive ascites, but a good general condition. B-mode ultrasound-guided HIPEC was performed in 22 patients with massive ascites and a poor general condition, and delayed CRS was performed once their general health condition improved (Table I).

Characteristics of ascites. Among the 68 patients with massive ascites, the amount of ascites ranged between 2,000 and $6,500 \mathrm{ml}$, with a mean amount of $3,532 \pm 379 \mathrm{ml}$, as confirmed by exploratory laparotomy drainage or by B-mode ultrasound-guided paracentesis drainage. Free cancer cells were observed in 57.4\% (39/68) of massive ascites (Table I).

In the good condition group, the amount of ascites ranged between 2,000 and $4,600 \mathrm{ml}$, with a mean amount of $2,650 \pm 232 \mathrm{ml}$, as confirmed by exploratory laparotomy drainage. Free cancer cells were observed in $56.5 \%(26 / 46)$ of massive ascites. In the poor condition group, the amount of ascites ranged between 3,200 and $6,500 \mathrm{ml}$, with a mean amount of $3,953 \pm 360 \mathrm{ml}$, as confirmed by paracentesis drainage guided by B-mode ultrasound. Free cancer cells were observed in 59.1\% (13/22) of massive ascites. The amount of ascites between the two groups was significantly different $(\mathrm{P}=0.010)$, whereas, no significant differences in the rate of free cancer cells positivity was observed between the two groups $(\mathrm{P}=0.080$; Table I).

Remission for malignant ascites. For 68 patients with massive ascites, all symptoms exhibited ascites regression after HIPEC, for a total ORR of $100 \%$, even for patients in the poor condition group. There were no cases of complete eradication of following HIPEC. There were no cases of unfeasible CRS among patients in both groups. No significant differences in ascites ORR rates were observed between the good and poor condition groups ( $\mathrm{P}=0.100$; Table I). Imaging and clinical examinations confirmed complete CRS in 58 patients. The 10 patients who underwent incomplete CRS exhibited no recurrence of malignant ascites during follow-up.

Changes in KPS score. In the present study, there were significant differences in KPS scores $(\mathrm{P}<0.01)$ between patients without/small malignant ascites and patients with massive malignant ascites prior to treatments. However, there were no significant differences in KPS scores between before treatment and after treatments $(\mathrm{P}=0.080)$.

Among the 1,125 patients with OC, but without/small ascites, KPS scores increased from $73.9 \pm 4.8$ before treatment to $74.1 \pm 5.3$ after treatment. There was a significant difference 
Table I. Analyses of the general condition of OC patients with malignant ascites treated with CRS + HIPEC.

\begin{tabular}{lccccc}
\hline & & \multicolumn{3}{c}{ Patients with ascites $(\mathrm{n}=68)$} & \\
\cline { 3 - 5 } Category & $\begin{array}{c}\text { Patients without } \\
\text { ascites }(\mathrm{n}=1,225)\end{array}$ & $\mathrm{CRS}+\mathrm{HIPEC}(\mathrm{n}=46)$ & $\mathrm{dCRS}+\mathrm{HIPEC}(\mathrm{n}=22)$ & Total (n=68) & P-value \\
\hline Ages, years old & $54.29 \pm 2.63$ & $54.47 \pm 2.28$ & $53.87 \pm 3.41$ & $54.16 \pm 2.87$ & 0.100 \\
Pretreatment KPS score, \% & $73.98 \pm 4.8$ & $52.8 \pm 3.1$ & $36.2 \pm 3.7$ & $48.9 \pm 2.3$ & 0.001 \\
Ascites, ml & - & $2650 \pm 332$ & $3953 \pm 360$ & $3532 \pm 349$ & 0.010 \\
FCC, $\%$ & - & $56.52(26 / 46)$ & $59.09(13 / 22)$ & $57.35(39 / 68)$ & 0.080 \\
PCI & $18 \pm 1.4(6-29)$ & $21 \pm 1.3(13-29)$ & $13 \pm 1.1(5-23)$ & $17 \pm 1.6(5-27)$ & 0.002
\end{tabular}

KPS scores, Karnofsky performance scale; FCC, free cancer cells; OC, ovarian cancer; PCI, peritoneal carcinomatosis index; CRS, cytoreductive surgery; HIPEC, hyperthermic intraperitoneal chemotherapy.

in KPS scores before treatments in patients without ascites and patients with massive ascites $(\mathrm{P}=0.001$; Table II). Among the 68 patients with OC and massive ascites, KPS scores significantly increased from $48.9 \pm 2.8$ before treatment to $74.1 \pm 4.3$ after treatment $(\mathrm{P}=0.001)$. In the good condition group, KPS scores significantly increased from $52.8 \pm 3.1$ before treatment to $73.9 \pm 4.7$ after treatment $(\mathrm{P}=0.001)$. In the poor condition group, KPS scores significantly increased from $36.2 \pm 3.7$ before treatment to $74.6 \pm 3.9$ after treatment $(\mathrm{P}=0.001)$. There were significant differences in KPS scores between the good and the poor condition groups at admission $(\mathrm{P}=0.001)$. However, no significant differences were noted in KPS scores following treatments $(\mathrm{P}=0.080$; Table II $)$.

CRS outcomes and PCI. Among the 1,225 patients with OC without/small ascites, complete CRS was achieved in 1,063 patients with a complete CRS success rate of $86.8 \%$ $(1,063 / 1,225)$; incomplete CRS was achieved in $162(13.2 \%$; $162 / 1,225$ ) patients without/small ascites (Table II). Among patients with ascites, complete CRS was achieved in 58 $(85.3 \%, 58 / 68)$ patients with primary OC and massive ascites, while incomplete CRS was achieved in 10 patients (14.71\%). There were no significant difference in the success of CRS between patients with ascites and those without/small ascites $(\mathrm{P}=0.100)$.

In the good condition group, complete CRS was achieved in $84.8 \%$ (39/46) of patients, and incomplete CRS was achieved in $15.2 \%(7 / 46)$. In the poor condition group, complete CRS was achieved in $86.4 \%(19 / 22)$ of patients and incomplete CRS achieved in $13.6 \%(3 / 22)$. There were no significant differences in the success of CRS between the two groups $(\mathrm{P}=0.080$; Table II).

Among the 1,225 patients without/small ascites, the mean PCI was $18.0 \pm 1.4$ (range, 6-29). Among the 68 patients with

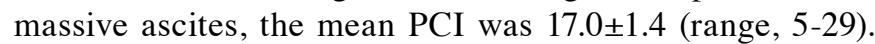
There were no significant differences in the mean PCI between patients without/small ascites and those with massive ascites $(\mathrm{P}=0.080$; Table I).

In the good condition group, the mean PCI was $21.0 \pm 1.3$ (range, 13-29) before HIPEC. In the poor condition group, the mean PCI was 13.0 \pm 1.1 (range, 5-23) as determined by CRS 2-4 weeks after HIPEC. There were significant differences in the mean PCI between the two groups $(\mathrm{P}=0.002$; Table I).
Adverse events due to HIPEC. Among the 1,225 patients with OC without/small ascites, adverse events due to HIPEC were observed in $101(8.9 \%, 101 / 1,225)$ patients, including $98(8.7 \%$, 98/1,225) patients with grade IV (CTCAE) bone marrow suppression (BMS) and three $(0.3 \%, 3 / 1,125)$ cases of severe renal failure (SRF) that required hemodialysis. Among the 68 patients with massive ascites, adverse events due to HIPEC were observed in nine $(13.2 \%, 9 / 68)$ patients, including six $(8.8 \%$, $6 / 68$ ) patients with grade IV (CTCAE) BMS and three $(4.4 \%$, $3 / 68$ ) cases of SRF that required hemodialysis. There were no significant differences in adverse events between patients with ascites and those without/small ascites $(\mathrm{P}=0.050$; Table II).

Grade IV BMS was observed in four $(8.7 \%, 4 / 46)$ patients in the good condition group, and in two $(9.1 \%, 2 / 22)$ patients in the poor condition group. SRF was observed in two $(4.4 \%$, $2 / 46)$ patients in the good condition group, and in one $(4.5 \%$, $2 / 22$ ) patient in the poor condition group. There were no significant differences in Grade IV BMS rates and SRF mobility between the two groups $(\mathrm{P}=0.050)$. The grade IV BMS were resolved after 1-3 weeks of granulocyte colony stimulating factor treatment and SRF cases were resolved by hemodialysis for $>1$ month. No other severe complications, including visceral injury, abdominal incision infection, or adhesive bowel obstruction, were observed following HIPEC (Table II).

Follow-up. The mean follow-up was 48 months (range, 7-98 months). The mean OS and DFS of patients without/small

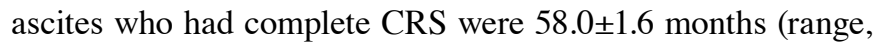
7-98 months) and 26.0 \pm 1.1 months (range, 8-52 months), respectively. The mean OS of patients without/small ascites

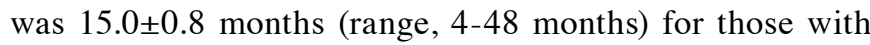
incomplete CRS. The median OS and DFS of patients with massive ascites who had complete CRS was $56.0 \pm 1.5$ months (range, 7-96 months), and 28.0 \pm 1.2 months (range, 8-49 months), respectively. The mean OS of patients with ascites was 15.0 \pm 1.0 months (range, 6-42 months) for those with incomplete CRS. There were no significant differences in mean OS and DFS between patients with ascites, and those without/small ascites $(\mathrm{P}=0.060)$, but there were significant differences in mean OS between patients with complete and incomplete CRS ( $\mathrm{P}=0.001$; data not shown).

In the good condition group, the mean OS and DFS of patients with massive ascites were 59.0 \pm 1.4 months (range, $8-89$ months) 
Table II. Analyses of the clinical effectiveness and side effects in OC patients with malignant ascites treated with HIPEC.

Patients with ascites $(n=68)$

Category Patients without ascites $(n=1,225) \quad$ CRS + HIPEC $(n=46) \quad$ HIPEC + dCRS $(n=22) \quad$ Total $(n=68) \quad$ P-value

\begin{tabular}{lccccc}
\hline SR, months & & & & & \\
OS & $59 \pm 1.6$ & $59 \pm 1.4$ & $56 \pm 1.6$ & $52 \pm 1.5$ & 0.060 \\
DFS & $25 \pm 1.1$ & $25 \pm 1.2$ & $27 \pm 1.3$ & $15.6 \pm 1.2$ & 0.070 \\
CRS, $\%$ & & & & \\
C-CRS & $86.31(971 / 1,125)$ & $84.78(39 / 46)$ & $86.36(19 / 22)$ & $85.29(58 / 68)$ & 0.080 \\
I-CRS & $13.68(154 / 1,125)$ & $15.22(4 / 46)$ & $13.64(3 / 22)$ & $14.71(10 / 68)$ & 0.090 \\
KPS score & $75.9 \pm 4.7$ & $73.9 \pm 4.7$ & $74.6 \pm 3.9$ & $74.1 \pm 4.3$ & 0.080 \\
Ascites CR, \% & 100 & 100 & 100 & 100 & 0.100 \\
Side effects, \% & & & & & \\
BMS & $8.71(98 / 1,125)$ & $8.70(4 / 46)$ & $9.09(2 / 22)$ & $8.82(6 / 68)$ & 0.050 \\
SRF & $0.26(3 / 1,125)$ & $4.34(2 / 46)$ & $4.54(1 / 22)$ & $4.41(3 / 68)$ & 0.010 \\
\hline
\end{tabular}

OS, overall survival; DFS disease-free survival; KPS scores, Karnofsky performance scale; CR, complete remission; dCRS, delayed cytoreductive surgery; C-CRS, complete CRS; I-CRS, incomplete CRS; BMS, bone marrow suppression; SFR, severe renal failure; HIPEC, hyperthermic intraperitoneal chemotherapy; OC, ovarian cancer.

and 25.0 \pm 1.2 months (range, 6-48 months), respectively. The mean OS of patients with massive ascites was 15.4 months (range, 7-49 months) for those with incomplete CRS. For patients with massive ascites in the poor condition group, the OS and DFS were 56.0 \pm 1.6 months (range, 6-83 months) and $27.0 \pm 1.3$ months (range, $8-46$ months), respectively. The median OS of patients with massive ascites was $15.0 \pm 1.1$ months (range, 6-46 months) for those with incomplete CRS. There were no significant differences in median OS and median DFS between the two groups (all $\mathrm{P}>0.05$ ), but there was a significant difference in OS between patients with complete and incomplete CRS patients $(\mathrm{P}=0.001$; Table II $)$.

\section{Discussion}

Ascites are frequently associated with PC originating from advanced OC, and PC is a typical feature of cancer spread in patients with primary advanced or recurrent OC, while distant metastases are rare $(1,2)$. CRS is the current standard therapy for patients with OC and peritoneal metastases. However, whether massive ascites affect the rate of complete CRS and prognosis for patients with primary OC under HIPEC treatment is not well defined. In the present study, optimal outcomes were achieved for patients with primary OC using HIPEC combined with CSR, as evidenced by the prolonged OS and DFS observed in the subgroup of patients treated with this combined therapy. These results suggest that massive ascites does not significantly affect the rate of complete CRS and prognosis. HIPEC is effective in increasing the rate of complete CRS. CRS is suitable for the majority of patients with primary OC and massive ascites, including those with poor conditions.

At the Intracelom Hyperthermic Perfusion Therapy Center, the first intention of CRS in patients with OC is always to achieve complete CRS. Eisenkop et al (38) suggested that a PCI of $<15$ is a determinant factor in achieving optimal cytoreduction and that a PCI of $>15$ would invariably preclude satisfactory surgical results. However, in the present study, the mean PCI was 17, and complete CRS was achieved in $>85 \%$ of patients with OC and malignant ascites, which is considerably higher compared with results previously published (38). Di Giorgio et al (44), demonstrated that in patients with ovarian carcinomatosis, a PCI of $>15$ does not invariably preclude satisfactory surgical results, which is supported by the results in the present study. Randle et al (3), suggested that the presence of malignant ascites significantly decreased the chances of achieving a complete CRS. In the present study, the success rate of complete CRS was $86.8 \%$ in patients without/small ascites, and $85.3 \%$ in patients with primary OC and malignant ascites. These results suggest that massive ascites does not affect the rate of complete CRS. In fact, a retrospective analysis of the study by Randle et al (3), revealed that there were numerous patients with digestive tract tumors, and that the rate of complete CRS in patients with OC and malignant ascites remained $100 \%$ (2). Therefore, the results presented by Randle et al (3), remain inconclusive for patients with OC.

Large ascites may affect respiratory and circulatory functions, making the tolerance of these patients to CRS and general anesthesia poor (4). B-mode ultrasound-guided HIPEC only requires local anesthesia, has limited effects on respiration and circulation compared with more invasive surgeries, and may offer palliative improvement by controlling ascites (4-7). In the present study, complete CRS was achieved in $86.4 \%$ of patients with poor health condition after HIPEC and there was no significant difference in the success rate of complete CRS compared with patients with a good health condition (84.8\%). This study suggests that with the improvement of the general condition after B-mode ultrasound-guided HIPEC, treatment with dCRS may allow outcomes that are similar to those of patients eligible for HIPEC during CRS. These results revealed that HIPEC may provide a possibility for complete CRS in patients with OC and ascites who are initially ineligible for CRS. 
Previous studies have suggested that killing microscopic tumors in the peritoneal cavity may be the major factor for HIPEC controlling malignant ascites from PC $(34,35,37)$. However, studies with laparoscopic HIPEC and B-mode ultrasound-guided HIPEC for the palliative control of malignant ascites, leaving the majority of tumor burden unaddressed following HIPEC, do not support this conclusion (3-9). In the present study, all patients with massive ascites exhibited ascites regression. The total objective remission rate was $100 \%$ following CRS and HIPEC in all groups, which included 22 patients in poor general condition in whom there were no cases of PC being completely eradicated after HIPEC confirmed by CRS, and 10 patients with incomplete CRS. Malignant ascites rarely relapse until PC progression and eventual mortality (4-7). Remission of ascites was completely independent of resection status of PC suggesting that it is more likely a function of HIPEC than CRS, or that HIPEC affects the ascites by an unidentified mechanism (3). Further studies are required to examine this issue.

Although CRS combined with adjuvant chemotherapy initially appears effective for advanced OC, insofar as high rates of patients achieve a good response, $~ 50 \%$ of patients relapse within 5 years and DFS times rarely exceed 18 months (44). The present study reported that a median DFS of 28 months and a median OS of 53.4 months is possible in patients with primary OC treated by HIPEC combined with CSR, even for patients with a poor general condition who are initially ineligible for CRS. In the present study, the median OS of patients with massive ascites from primary OC who underwent complete CRS was 56 months, while incomplete CRS resection led to an OS of 15 months. These results suggest that CRS combined with HIPEC may prolong the median DFS and OS of patients with primary OC and massive ascites, but only in cases in which a successful complete CRS is achieved. In addition, patients with $\mathrm{OC}$ and malignant ascites with incomplete CRS exhibit a poor prognosis, as do patients without/small malignant ascites treated by incomplete CRS.

The procedures presented in the current study have no unacceptable toxic effects, except for grade IV BMS and SRF (4-7). In the present study, no significant difference was identified between patients with ascites and without/small ascites in grade IV BMS (8.7 vs. 8.8\%), but the SRF of patients with massive ascites was significant higher compared with that in patients without/small ascites (0.3 vs. $4.4 \%)$. At present, there is a lack of understanding of the adverse effects of heat stress combined with chemotherapeutic drugs.

The present study is not without limitations. The number of patients with massive ascites was relatively small, limiting the conclusions and in-depth analyses. Certain patients may have been lost to follow-up, introducing a bias. Finally, the patients were selected according to a criterion, introducing another bias. Multicenter randomized controlled trials are necessary to assess adequately the effect of HIPEC in patients with OC and ascites.

In conclusion, ascites does not appear to affect the rate of complete CRS and prognosis of patients with massive ascites following HIPEC. Patients with OC and massive ascites still have relatively high rates of complete CRS with a good prognosis. CRS+HIPEC may prolong the median DFS and OS of patients with primary $\mathrm{OC}$, and massive ascites.

\section{Acknowledgements}

Not applicable.

\section{Funding}

The present study was supported by the Guangzhou Key Medical Discipline Construction Project (grant no. 2017), Guangdong Science and Technology Plan Project (grant no. 20160918), Guangzhou Science Technology and Innovation Commission (grant no. 2014Y2-00152), and Guangzhou Science Technology and Innovation Commission (grant no. 2014Y2-00548).

\section{Availability of data and materials}

The datasets used and/or analyzed during the present study are available from the corresponding author on reasonable request.

\section{Authors' contributions}

MB conceived and coordinated the study, designed, performed and analyzed the experiments, wrote the paper. MB and HL carried out the data collection, data analysis, and revised the paper. MB, HL, XZ, ZY, SW, YW, YG and SC carried out the data collection, data analysis and reviewed the results. All authors approved the final version of the manuscript.

\section{Ethics approval and consent to participate}

The present study was approved by the Medical Ethics Committee of the Cancer Hospital of Guangzhou Medical College (approval no. GZYY2006-8-20). Written informed consent was obtained from all patients.

\section{Patient consent for publication}

Patients provide consent for the publication of images.

\section{Competing interests}

The authors declare that they have no competing interests.

\section{References}

1. Coleman RL, Monk BJ, Sood AK and Herzog TJ: Latest research and treatment of advanced-stage epithelial ovarian cancer. Nat Rev Clin Oncol 10: 211-24, 2013.

2. Sato $\mathrm{S}$ and Itamochi H: Neoadjuvant chemotherapy in advanced ovarian cancer: Latest results and place in therapy. Ther Adv Med Oncol 6: 293-304, 2014

3. Randle RW, Swett KR, Swords DS, Shen P, Stewart JH, Levine EA and Votanopoulos KI: Efficacy of cytoreductive surgery with hyperthermic intraperitoneal chemotherapy in the management of malignant ascites. Ann Surg Oncol 21: 1474-1479, 2014.

4. Ba M, Long H, Zhang X, Tang Y, Wu Y, Yu F, Wang S and Cui S: Different sequential approaches of cytoreductive surgery and hyperthermic intraperitoneal chemotherapy in treating ovarian cancer with malignant ascites. J Cancer Res Clin Oncol 140: 1497-1506, 2014.

5. Ba MC, Cui SZ, Lin SQ, Tang YQ, Wu YB, Wang B and Zhang XL: Chemotherapy with laparoscope-assisted continuous circulatory hyperthermic intraperitoneal perfusion for malignant ascites. World J Gastroenterol. 16: 1901-1907, 2010. 
6. Ba MC, Long H, Cui SZ, Tang YQ, Wu YB, Zhang XL, Tang HS and Bai SX: Multivariate comparison of B-ultrasound guided and laparoscopic continuous circulatory hyperthermic intraperitoneal perfusion chemotherapy for malignant ascites. Surg Endosc 27: 2735-2743, 2013.

7. Cui S, Ba M, Tang Y, Liu J, Wu Y, Wang B, Zhang X, Tang H and Zhong S: B ultrasound-guided hyperthermic intraperitoneal perfusion chemotherapy for the treatment of malignant ascites. Oncol Rep 28: 1325-1331, 2012.

8. Facchiano E, Scaringi S, Kianmanesh R, Sabate JM, Castel B, Flamant Y, Coffin B and Msika S: Laparoscopic hyperthermic intraperitoneal chemotherapy (HIPEC) for the treatment of malignant ascites secondary to unresectable peritoneal carcinomatosis from advanced gastric cancer. Eur J Surg Oncol 34 $154-158,2008$

9. Garofalo A, Valle M, Garcia J and Sugarbaker PH: Laparoscopic intraperitoneal hyperthermic chemotherapy for palliation of debilitating malignant ascites. Eur J Surg Oncol 32: 682-685, 2006.

10. Parson EN, Lentz S, Russell G, Shen P, Levine EA and Stewart JH 4th: Outcomes after cytoreductive surgery and hyperthermic intraperitoneal chemotherapy for peritoneal surface dissemination from ovarian neoplasms. Am J Surg 202: 481-486, 2011.

11. Liu Y, Endo Y, Fujita T, Ishibashi H, Nishioka T, Canbay E, Li Y, Ogura S and Yonemura Y: Cytoreductive surgery under aminolevulinic acid-mediated photodynamic diagnosis plus hyperthermic intraperitoneal chemotherapy in patients with peritoneal carcinomatosis from ovarian cancer and primary peritoneal carcinoma: Results of a phase I trial. Ann Surg Oncol 21: 4256-4262, 2014.

12. Chia CS, Tan WJ, Wong JF, Tan GH, Lim C, Wang W, Sin EI, Tham CK, Soo KC and Teo MC: Quality of life in patients with peritoneal surface malignancies after cytoreductive surgery and hyperthermic intraperitoneal chemotherapy. Eur J Surg Oncol 40: 909-916, 2014

13. Tan WJ, Wong JF, Chia CS, Tan GH, Soo KC and Teo MC: Quality of life after cytoreductive surgery and hyperthermic intraperitoneal chemotherapy: An Asian perspective. Ann Surg Oncol 20: 4219-4223, 2013.

14. Glehen O, Gilly FN, Arvieux C, Cotte E, Boutitie F, Mansvelt B, Bereder JM, Lorimier G, Quenet F and Elias D; Association Française de Chirurgie: Peritoneal carcinomatosis from gastric cancer: A multi-institutional study of 159 patients treated by cytoreductive surgery combined with perioperative intraperitoneal chemotherapy. Ann Surg Oncol 17: 2370-2377, 2010.

15. Tabrizian P, Shrager B, Jibara G, Yang MJ, Romanoff A, Hiotis S, Sarpel U and Labow DM: Cytoreductive surgery and hyperthermic intraperitoneal chemotherapy for peritoneal carcinomatosis: Outcomes from a single tertiary institution. J Gastrointest Surg 18: 1024-1031, 2014

16. Graziosi L, Marino E and Donini A: Reply to 'Peritoneal carcinomatosis in patients with gastric cancer, and the role for surgical resection, cytoreductive surgery, and hyperthermic intraperitoneal chemotherapy'. Am J Surg 208: 158-159, 2014.

17. Scaringi S, Kianmanesh R, Sabate JM, Facchiano E, Jouet P, Coffin B, Parmentier G, Hay JM, Flamant Y and Msika S: Advanced gastric cancer with or without peritoneal carcinomatosis treated with hyperthermic intraperitoneal chemotherapy: A single western center experience. Eur J Surg Oncol 34: 1246-1252, 2008.

18. Zanon C, Bortolini M, Chiappino I, Simone P, Bruno F, Gaglia P, Airoldi M, Deriu L and Mashiah A: Cytoreductive surgery combined with intraperitoneal chemohyperthermia for the treatment of advanced colon cancer. World J Surg 30: 2025-2032, 2006.

19. Virzi S, Iusco D, Baratti D, Bonomi S, Grassi A, Kusamura S and Deraco M: Pilot study of adjuvant hyperthermic intraperitoneal chemotherapy in patients with colorectal cancer at high risk for the development of peritoneal metastases. Tumori 99: 589-595, 2013.

20. Deraco M, Baratti D, Inglese MG, Allaria B, Andreola S, Gavazzi $C$ and Kusamura S: Peritonectomy and intraperitoneal hyperthermic perfusion (IPHP): A strategy that has confirmed its efficacy in patients with pseudomyxoma peritonei. Ann Surg Oncol 11: 393-398, 2004

21. Kuijpers AM, Mehta AM, Aalbers AG, van Driel WJ, Boot $H$ and Verwaal VJ: Treatment of ovarian metastases of colorectal and appendiceal carcinoma in the era of cytoreductive surgery and hyperthermic intraperitoneal chemotherapy. Eur J Surg Oncol 40: 937-942, 2014
22. Ansaloni L, Agnoletti V, Amadori A, Catena F, Cavaliere D, Coccolini F, De Iaco P, Di Battista M, Framarini M, Gazzotti F, et al: Evaluation of extensive cytoreductive surgery and hyperthermic intraperitoneal chemotherapy (HIPEC) in patients with advanced epithelial ovarian cancer. Int J Gynecol Cancer 22: 778-785, 2012

23. Pavlov MJ, Kovacevic PA, Ceranic MS, Stamenkovic AB, Ivanovic AM and Kecmanovic DM: Cytoreductive surgery and modified heated intraoperative intraperitoneal chemotherapy (HIPEC) for advanced and recurrent ovarian cancer-12-year single center experience. Eur J Surg Oncol 35: 1186-1191, 2009.

24. Deraco M, Kusamura S, Virzi S, Puccio F, Macri A, Famulari C, Solazzo M, Bonomi S, Iusco DR and Baratti D: Cytoreductive surgery and hyperthermic intraperitoneal chemotherapy as upfront therapy for advanced epithelial ovarian cancer: multi-institutional phase-II trial. Gynecol Oncol 122: 215-220, 2011.

25. Tentes AA, Kakolyris S, Kyziridis D and Karamveri C: Cytoreductive surgery combined with hyperthermic intraperitoneal intraoperative chemotherapy in the treatment of advanced epithelial ovarian cancer. J Oncol 2012: 358341, 2012

26. Spiliotis J, Vaxevanidou A, Sergouniotis F, Lambropoulou E, Datsis A and Christopoulou A: The role of cytoreductive surgery and hyperthermic intraperitoneal chemotherapy in the management of recurrent advanced ovarian cancer: A prospective study. J BUON 16: 74-79, 2011.

27. Safra T, Grisaru D, Inbar M, Abu-Abeid S, Dayan D, Matceyevsky D, Weizman A and Klausner JM: Cytoreduction surgery with hyperthermic intraperitoneal chemotherapy in recurrent ovarian cancer improves progression-free survival, especially in BRCA-positive patients-a case-control study. J Surg Oncol 110: 661-665, 2014

28. Rettenmaier MA, Mendivil AA, Abaid LN, Brown Iii JV, Wilcox AM and Goldstein BH: Consolidation hyperthermic intraperitoneal chemotherapy and maintenance chemotherapy following laparoscopic cytoreductive surgery in the treatment of ovarian carcinoma. Int J Hyperthermia 31: 8-14, 2015.

29. Chiva LM and Gonzalez-Martin A: A critical appraisal of hyperthermic intraperitoneal chemotherapy (HIPEC) in the treatment of advanced and recurrent ovarian cancer. Gynecol Oncol 136: $130-5,2015$

30. Munoz-Casares FC, Rufian S, Rubio MJ, Diaz CJ, Diaz R, Casado A, Arjona A, Muñoz-Villanueva MC and Muntané J: The role of hyperthermic intraoperative intraperitoneal chemotherapy (HIPEC) in the treatment of peritoneal carcinomatosis in recurrent ovarian cancer. Clin Transl Oncol 11: 753-759, 2009.

31. Le Brun JF, Campion L, Berton-Rigaud D, Lorimier G, Marchal F, Ferron G, Oger AS, Dravet F, Jaffre I and Classe JM: Survival benefit of hyperthermic intraperitoneal chemotherapy for recurrent ovarian cancer: A multi-institutional case control study. Ann Surg Oncol 21: 3621-3627, 2014.

32. Rettenmaier MA, Mendivil AA, Abaid LN, Brown JV III, Micha JP, Wilcox AM and Goldstein BH: The feasibility of administering varying high-dose consolidation hyperthermic intraperitoneal chemotherapy with carboplatin in the treatment of ovarian carcinoma. Arch Gynecol Obstet 291: 1381-1386, 2015

33. Cascales Campos P, Gil J and Parrilla P: Morbidity and mortality outcomes of cytoreductive surgery and hyperthermic intraperitoneal chemotherapy in patients with primary and recurrent advanced ovarian cancer. Eur J Surg Oncol 40: 970-975, 2014.

34. Cascales Campos PA, Gil Martinez J, Galindo Fernandez PJ, Gil Gomez E, Martinez Frutos IM and Parrilla Paricio P: Perioperative fast track program in intraoperative hyperthermic intraperitoneal chemotherapy (HIPEC) after cytoreductive surgery in advanced ovarian cancer. Eur J Surg Oncol 37: 543-548, 2011.

35. Cascales-Campos PA, Gil J,Feliciangeli E, Gil E, Gonzalez-Gil A, Lopez V, Ruiz-Pardo J, Nieto A, Parrilla JJ and Parrilla P: The role of hyperthermic intraperitoneal chemotherapy using paclitaxel in platinum-sensitive recurrent epithelial ovarian cancer patients with microscopic residual disease after cytoreduction. Ann Surg Oncol 22: 987-993, 2015.

36. Cascales-Campos PA, Gil J, Gil E, Feliciangeli E, Gonzalez-Gil A, Parrilla JJ and Parrilla P: Treatment of microscopic disease with hyperthermic intraoperative intraperitoneal chemotherapy after complete cytoreduction improves disease-free survival in patients with stage IIIC/IV ovarian cancer. Ann Surg Oncol 21: 2383-2389, 2014 .

37. Coccolini F, Campanati L, Catena F, Ceni V, Ceresoli M, Jimenez Cruz J, Lotti M, Magnone S, Napoli J, Rossetti D, et al: Hyperthermic intraperitoneal chemotherapy with cisplatin and paclitaxel in advanced ovarian cancer: A multicenter prospective observational study. J Gynecol Oncol 26: 54-61, 2015. 
38. Eisenkop SM, Spirtos NM, Friedman RL, Lin WC, Pisani AL and Perticucci S: Relative influences of tumor volume before surgery and the cytoreductive outcome on survival for patients with advanced ovarian cancer: A prospective study. Gynecol Oncol 90: 390-396, 2003.

39. Coccolini F, Gheza F, Lotti M, Virzi S, Iusco D, Ghermandi C, Melotti R, Baiocchi G, Giulini SM, Ansaloni L and Catena F: Peritoneal carcinomatosis. World J Gastroenterol 19: 6979-6994, 2013.

40. Munoz-Casares FC, Rufian S, Rubio MJ, Lizarraga E, Diaz-Iglesias C, Aranda E, Ciria R, Muntané J, Barrios P, Torres-Melero J, et al: Treatment of peritoneal carcinomatosis from ovarian cancer. Present, future directions and proposals. Clin Transl Oncol 9: 652-662, 2007.
41. Fagotti A, Gallotta V, Romano F, Fanfani F, Rossitto C, Naldini A, Vigliotta M and Scambia G: Peritoneal carcinosis of ovarian origin. World J Gastrointest Oncol 2: 102-108, 2010.

42. Prat J; FIGO Committee on Gynecologic Oncology: Staging classification for cancer of the ovary, fallopian tube, and peritoneum. Int J Gynaecol Obstet 124: 1-5, 2014.

43. Sugarbaker PH: Peritonectomy procedures. Ann Surg 221: 29-42, 1995

44. Di Giorgio A, Naticchioni E, Biacchi D, Sibio S, Accarpio F, Rocco M, Tarquini S, Di Seri M, Ciardi A, Montruccoli D and Sammartino P: Cytoreductive surgery (peritonectomy procedures) combined with hyperthermic intraperitoneal chemotherapy (HIPEC) in the treatment of diffuse peritoneal carcinomatosis from ovarian cancer. Cancer 113: 315-325, 2008. 